\title{
Genetic analyses of pyloric stenosis suggesting a specific maternal effect*
}

\author{
K. K. KIDD and M. A. SPENCE \\ From the Department of Human Genetics, Yale University, New Haven CT 06510; and \\ the Departments of Psychiatry and Biomathematics, UCLA, Los Angeles CA 90024, USA
}

Summary. Data on pyloric stenosis are analysed by multiple threshold methods that incorporate the sex effect. The polygenic model of inheritance is rejected; the single major locus model can only account for $37 \%$ of the cases having a genetic aetiology, requiring an environmental effect to account for the remainder of the cases. A maternal-fetal interaction for gastrin production and sensitivity is postulated to explain all the existing data. The empirical risk figures given are considered to be the most accurate basis for genetic counselling until additional studies provide a sound biological basis for a quantitative genetic model.

The multifactorial (MF) or polygenic threshold mode of inheritance was introduced by Crittenden (1961) and Falconer (1965). This model was proposed as the mode of inheritance for pyloric stenosis, a trait that does not display simple Mendelian patterns (Falconer, 1965). The proposal was supported by the report of Carter and Evans (1969) in which they analysed extensive pyloric stenosis data for estimates of heritability.

Generalized single major locus (SML) models have been developed for other traits which show no clear Mendelian pattern (Campbell and Elston, 1971; Kidd and Cavalli-Sforza, 1973). These interconvertible SML models include incomplete penetrance for the three genotypes; autosomal dominant and recessive modes of inheritance are special cases. The assumptions of the MF and SML models differ. The MF model assumes many segregating loci each of small effect, with both transmitted and individual environmental factors possible; the SML model assumes only one twoallele locus with no other transmitted factors but

\footnotetext{
Received 17 June 1975.

* This research supported in part by: University of California at Los Angeles; Mental Retardation Program and Child Psychiatry Program, NPI, UCLA, MCH-927; Interdisciplinary Training in Mental Retardation, HD-04612; Mental Retardation Center, UCLA, HD-00345; Research Training in Mental Retardation, HD-05615; Developmental Biology in Mental Retardation; and a research grant (to K. K. Kidd) from the P.H.S. National Institute of Neurological Diseases and Stroke, NS-11786.
} with random enviromental variation acting inde- $\square$ pendently within families. The MF and SML models have been shown to be indistinguishable as explanations for many types of family data (Elstono and Campbell, 1970; Smith, 1971; Kidd and用 Cavalli-Sforza, 1973) and are not hypotheses that can be tested when only one threshold is presento응 (James, 1971; Reich, James, and Morris, 1972). The incorporation of two thresholds into the models, thereby defining a mild and severe form of the trait does permit goodness-of-fit tests and, hence, the possibility of distinguishing between the modelso (Reich et al, 1972). Kidd, Reich, and Kessler 3 (1973) have shown that the same statistical benefitsi occur when a sex effect exists and is treated as two thresholds, a lower one for the commonly affected sex and a higher one for the less commonly affected? sex.

There are no clinically recognized mild and severe forms of pyloric stenosis, but males are four timeso more commonly affected than females (Carter, N 1974). Therefore, the $M F$ and SML models, representing the extreme alternatives of the tradi- $-\omega$ tional models of human genetics, generalized to incorporate incomplete penetrance and a sex effect in ac quantitative manner, are applied in this paper tos data on pyloric stenosis for the purpose of testing whether either model is an acceptable explanation for the inheritance of this condition. 


\section{Subjects and methods}

The pooled data that are analysed in this paper come from the following sources: the data reported by Carter and Evans (1969) incorporating the updated figures of Carter (1974); the family data of McKeown, MacMahon, and Record (1951a); and the Caucasian families in the Hawaiian study of Campbell (1970). The total sample consists of data for 2903 index cases, 882 offspring, and 2253 sibs. In addition, data were assembled on 92 pairs of monozygotic $(\mathrm{MZ})$ twins; dizygotic twins $(\mathrm{DZ})$ are included in the analyses as sibs. The $M Z$ twins include 77 pairs reported by Powell and Carter (1951) and 75 pairs taken from other studies and from single published case reports (Welsh, 1951; Metrakos, 1953; Lee and Martmer, 1962; William, 1963; Campbell, 1970). Sixty-two of 72 male $M Z$ pairs were concordant for pyloric stenosis; 14 of 20 female $M Z$ pairs were concordant. The recent report of pyloric stenosis in identical triplets (Spitz, 1974) was not included in the analyses. General incidence figures of 48 in 10000 males and 12 in 10000 females were included in the analyses to restrict the solutions found to those with general incidences close to the generally accepted values for Caucasians (Carter, 1974; Campbell, 1970). Table I gives the observed frequencies of affected relatives of probands in the pooled data.

The method of analysis incorporating sex effect into the MF and SML models is used (Kidd et al, 1973).* The analysis for either model consists of varying parameter values until that set is found which minimizes the $\chi^{2}$ value as a measure of difference between observed and expected incidences among relatives. The MF model has four parameters: the general incidences for males and

* Copies of the FORTRAN IV programs for these analyses, MFSEX, and SMLSEX are available from K. Kidd.

\section{TABLE I}

COMPARATIVE RISKS FOR PYLORIC STENOSIS EXCLUDING MZ TWIN DATA

\begin{tabular}{|c|c|c|c|}
\hline \multirow{2}{*}{ Relatives } & & \multicolumn{2}{|c|}{ Probands } \\
\hline & & Male & Fenuale \\
\hline Brothers & $\begin{array}{l}\text { Obs. } \\
\text { MF } \\
\text { SML }\end{array}$ & $\begin{array}{l}0.061, \mathrm{~N}=826 \\
0.075(2.421) \\
0.060(0.001)\end{array}$ & $\begin{array}{l}0.106, N=359 \\
0.109(0.040) \\
0.124(1.096)\end{array}$ \\
\hline Sisters & $\begin{array}{l}\text { Obs. } \\
\text { MF } \\
\text { SML }\end{array}$ & $\begin{array}{l}0.024, \mathrm{~N}=757 \\
0.025(0.042) \\
0.024(0.012)\end{array}$ & $\begin{array}{l}0.042, \mathrm{~N}=311 \\
0.040(0.030) \\
0.051(0.594)\end{array}$ \\
\hline Sons & $\begin{array}{l}\text { Obs. } \\
\text { MF } \\
\text { SML }\end{array}$ & $\begin{array}{l}0.055, N=346 \\
0.075(1.973) \\
0.060(0.175)\end{array}$ & $\begin{array}{l}0.194, \mathrm{~N}=103 \\
0.109(7.661) \\
0.124(4.659)\end{array}$ \\
\hline Daughters & $\begin{array}{l}\text { Obs. } \\
\text { MF } \\
\text { SML }\end{array}$ & $\begin{array}{l}0.024, N=337 \\
0.025(0.020) \\
0.024(0.006)\end{array}$ & $\begin{array}{l}0.073, N=96 \\
0.040(2.735) \\
0.051(0.911)\end{array}$ \\
\hline $\begin{array}{l}\text { General } \\
\text { incidence }\end{array}$ & $\begin{array}{l}\text { Obs. } \\
\text { MF } \\
\text { SML }\end{array}$ & $\begin{array}{l}0.0048 \\
0.0048(0) \\
0.0051(0.149)\end{array}$ & $\begin{array}{l}0.0012 \\
0.0011(0.091) \\
0.0010(0.412)\end{array}$ \\
\hline
\end{tabular}

Observed incidences and predicated frequencies of affected individuals for the multifactorial (MF) and single major locus (SML) models are indicated for pyloric stenosis. $\chi^{2}$ values obtained from the $2 \times 2$ table analysis are indicated in parentheses. Sample sizes are given for each category $(N)$, see text for sources of data. females, and the genetic correlations between sibs and between parent and offspring. The SML model has five parameters: the gene frequency, the environmental standard deviation, one threshold for each sex, and the position of the genotypic mean for heterozygotes relative to the means for the two homozygous genotypes. The generalized model and parameters are illustrated in the Fig. These parameters are identical to those in Kidd and Cavalli-Sforza (1973) and Kidd (1975) except for the addition of the second threshold.

\section{Results}

The initial analyses, including available data on $M Z$ twins, resulted in $\chi^{2}$ values that were exceedingly large:

$$
\chi_{8}^{2}=28, \mathrm{P}<0.001 \text {, and } \chi_{7}^{2}=250, \mathrm{P}<0.001 \text {, }
$$

for the MF and SML models, respectively. The predicted frequencies for $M Z$ twins were always considerably less than the reported frequencies. Because we felt this might be reflecting ascertainment bias or incorrect assignment of concordance as discussed by Smith (1974), the data on $M Z$ twins were excluded from further consideration in all the analyses.

Subsequent analysis under the MF model yields estimates of 0.0048 and 0.0011 for the male and

TABLE II

THE SML SOLUTION

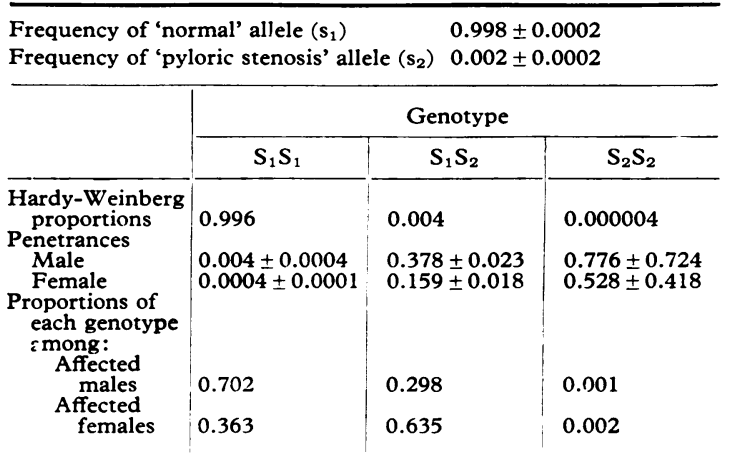

Liability scale varameters

$$
\begin{aligned}
& \varepsilon=0.580 \pm 0.009 \\
& \mathrm{~T}_{m}=1.560 \pm 0.029 \\
& \mathrm{~T}_{f}=1.960 \pm 0.043 \\
& \mathrm{M}_{1}=1.380 \pm 0.024 \\
& \chi_{5}^{2}=8.015, \mathrm{P} \sim 0.15
\end{aligned}
$$

Minimum $\chi^{2}$ estimates for the five parameters of the single major locus model are given with the standard error of the estimate: $q=$ gene frequency of the pyloric stenosis allele, $\varepsilon=$ the environmental standard deviation, $T_{m}=$ threshold for males, $T_{f}=$ threshold tor females, $M_{1}=$ the genotypic value of the heterozygotes. The last four parameters are given on a liability scale arbitrarily fixed by setting the means of the homozygotes, $S_{1} S_{1}$ and $S_{2} S_{2}$, to 0 and 2 , respectively (see Fig). The $x^{2}$ for goodness of fit is taken from the predicted frequencies given in Table I. 


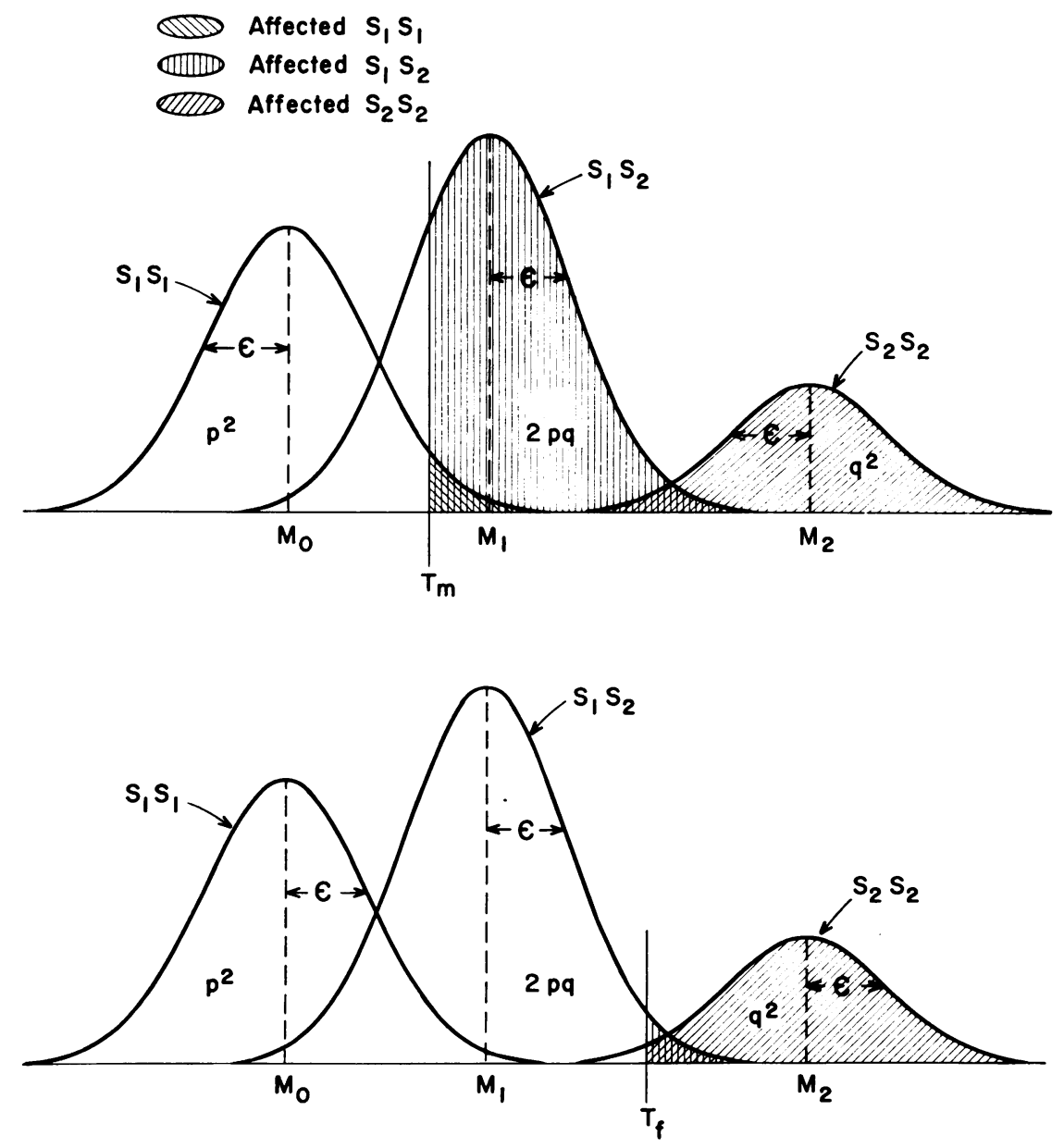

Fig. A graphical representation of the general SML model with sex effect. The upper distribution represents the more commonly affected sex (males in the case of pyloric stenosis); the lower distribution represents the less commonly affected sex (females). All parameters are the same for both sexes except for the thresholds. The liability scale is arbitrarily fixed by assigning values to the genotype means of the homozygotes, $\mathrm{M}_{0}$ and $\mathrm{M}_{2}$. The five parameters are then the gene frequency, $q$, the environmental standard deviation $\varepsilon$, the positions of the two thresholds, $T_{m}$ and $T_{f}$ and the position of the heterozygote mean, $M_{1}$.

female general incidences, respectively. The estimate of the liability correlation coefficient for sibs and for parents-offspring is 0.442 . The predicted frequencies for each class, based on those estimates, are given in Table I. The comparison of expected to observed yields a $\chi_{6}^{2}=15.01, P \sim 0.02$. Comparable estimates of parameters for the SML model are given in Table II. Predicted frequencies are again listed in Table I. The $\chi_{5}^{2}=8.02$ was not significant, $\mathbf{P} \sim 0.15$. Neither set of results is significantly altered by exclusion of the general incidence figures from the analyses.

\section{Discussion}

The classical MF model is excluded by these analyses $(P \sim 0.02)$. The SML model, which cannot be excluded by these data, postulates a very rare, essentially dominant, allele. However, $70 \%$ of affected males and $36 \%$ of affected females would lack this allele and represent environmentally caused phenocopies. Thus, neither genetic model seems a satisfactory explanation for the majority of the occurrences of pyloric stenosis.

Inspection of the $2 \times 2 \chi^{2}$ values in Table I shows that the greatest discrepancies between observed 
and expected for both models occur for the incidences of offspring, particularly sons, of women who had themseves been affected as infants. Indeed, the incidence among offspring of affected females is significantly greater than that among sibs of females $\left(\chi_{1}^{2}=6.7, P<0.01\right)$, confirming the same observation by Carter (1963) based on about half of the present data. Neither of our models can explain this difference; it suggests a specific maternal effect restricted to those women who were themselves affected. Previous studies have attempted to define a maternal effect such as age (McKeown, MacMahon, and Record, 1951b) but found none. Dodge (1975) reports an association with breast feeding. In these studies, the specific maternal effect suggested here would not have been detectable because such a small fraction of affected individuals are born to women who are affected.

A maternal physiological characteristic, possibly related to human pyloric stenosis, has been investigated in dogs. These experimental studies have indicated that raised levels of maternal gastrin may produce hypertrophied pyloric tumours in fetal and newborn dogs (Dodge, 1970). This is interpreted as interaction between maternal gastrin and the fetus or newborn, since the small gastrin molecule has been shown to cross the placenta in experimental work with dogs (Bruckner, Snow, and Fonkalsrud, 1970). In human mothers, Dodge (1972) reports increased stress factors in the first pregnancy and suggests that this would result in altered physiology, perhaps reflected in increased gastrin secretions. This evidence combined with our analyses suggests a model for the genetics of pyloric stenosis in man: hypertrophy of the pylorus results from the interaction of maternal gastrin concentrations and fetal sensitivity to the concentration.

Because $M Z$ twins share both an identical genotype and an essentially identical intrauterine environment, the postulated maternal effect predicts that concordances would be significantly raised over those expected from the models analysed. Though $M Z$ data were originally excluded because a serious sampling bias was suspected, in retrospect, the 'raised' concordance might be real. This model also provides an explanation for the statistically significant increase in firstborn infants affected with pyloric stenosis (McKeown et al, 1951b), as suggested by Dodge (1972). It would also explain, assuming Dodge is correct, the confirmed finding of raised frequency in offspring of affected females. Females who were themselves affected represent a selected population with, on average, significantly raised gastrin concentrations, resulting in an increased frequency of affected offspring.
Both the MF and SML models could be modified in several ways to incorporate a specific maternal effect of the sort postulated. However, two 'traits', possibly genetically independent, are actually involved: increased gastrin secretion by certain women, and a greater fetal sensitivity to maternal gastrin concentrations. Either alone could be the major factor, or the two could interact in a variety of ways. Given only borderline exclusion of the MF model and non-exclusion of the SML model, any similar model with a greater number of parameters will be difficult, if not impossible, to exclude. Thus, little can be gained by further elaboration of the models without a sound biological basis for additional parameters. Experimental designs could be followed to collect relevant biological data. Given the exclusion of the MF model, the risk figures given by Bonaiti-Pellie and Smith (1974) must be considered crude approximations. Even risk figures based on the SML model are suspect. For the present, genetic counselling for simple family situations should rely on the empiric risks (Table I).

\section{REFERENCES}

Bonaiti-Pellie, C. and Smith, C. (1974). Risk tables for genetic counselling in some common malformations. Fournal of Medical Genetics, 11, 374-377.

Bruckner, W. L., Snow, H. D., and Fonkalsrud, E. W. (1970). Gastric secretion in the canine fetus following maternal stumulation: experimental studies on placental transfer of insulin, histamine and gastrin. Surgery, 67, 360-363.

Campbell, M. A. (1970). Studies on quasi-continuity. Dissertation, University of Hawaii, Honolulu. Dissertation Abstracts, 31, order no. 70-19,506.

Campbell, M. A. and Fiston, R. C. (1971). Relatives of probands: models for preliminary genetic analysis. Annals of Human Genetics, 35, 225-235.

Carter, C. O. (1963). The genetics of common malformations. In Second International Conference on Congenital Malformations, pp. 306-313. Compiled, Edited, and Published by The International Medical Congress Ltd., New York.

Carter, C. O. (1974). Recurrence risk of common congenital malformations. Practitioner, 213, 667-674.

Carter, C. O. and Evans, K. A. (1969). Inheritance of congenital pyloric stenosis. Fournal of Medical Genetics, 6, 233-254.

Crittenden, L. B. (1961). Interpretation of familial aggregation based on multiple genetic and environmental factors. Annals of the New York Academy of Sciences, 91, 769-780.

Dodge, J. A. (1970). Production of duodenal ulcers and hypertrophic pyloric stenosis by administration of pentagastrin to pregnant and newborn dogs. Nature (London), 225, 284-285.

Dodge, J. A. (1972). Psychosomatic aspects of infantile pyloric stenosis. Fournal of Psychosomatic Research, 16, 1-5.

Dodge, J. A. (1975). Infantile hypertrophic pyloric stenosis in Belfast, 1957-1969. Archives of Disease in Childhood, 50, 171-178.

Elston, R. C. and Campbell, M. A. (1970). Schizophrenia: evidence for the major gene hypothesis. Behavior Genetics, 1, 3-10.

Falconer, D. S. (1965). The inheritance of liability to certain diseases, estimated from the incidence among relatives. Annals of Human Genetics, 29, 51-76.

James, J. W. (1971). Frequency in relatives for an all or none trait. Annals of Human Genetics, 35, 47-50.

Kidd, K. K. (1975). On the possible magnitudes of selective forces maintaining schizophrenia in the population. In Genetic Research in Psychiatry, pp. 135-145. Ed. by R. R. Fieve, D. Rosenthal, and $\mathrm{H}$. Brill. The Johns Hopkins University Press, Baltimore.

Kidd, K. K. and Cavalli-Sforza, L. L. (1973). An analysis of the genetics of schizophrenia. Social Biology, 20, 254-265. 
Kidd, K. K., Reich, T., and Kessler, S. (1973). A genetic analysis of stuttering suggesting a single major locus (abstract). Genetics, 74, s137.

Lee, R. K. and Martmer, E. E. (1962). Pyloric stenosis in fraternal twins. Harper Hospital Bulletin, 20, 244-245.

Mckeown, T., MacMahon, B., and Record, R. G. (1951a). The familial incidence of congenital pyloric stenosis. Annals of Human Genetics, 16, 260-281.

McKeown, T., MacMahon, B., and Record, R. G. (1951b). The incidence of congenital pyloric stenosis related to birth rank and maternal age. Annals of Human Genetics, 16, 249-259.

Metrakos, J. D. (1953). Congenital hypertrophic pyloric stenosis in twins. Archives of Disease in Childhood, 27, 351-358.

Powell, B. W. and Carter, C. O. (1951). Pyloric stenosis in twins. Archives of Disease in Childhood, 26, 45-49.
Reich, T., James, J. W., and Morris, C. A. (1972). The use of $\frac{\mathbb{D}}{\frac{1}{2}}$ multiple thresholds in determining the mode of transmission of $\mathbb{D}$ semi-continuous traits. Annals of Human Genetics, 36, 163-184.

Smith, C. (1971). Discrimination between different modes of inheritance in genetic disease. Clinical Genetics, 2, 303-314.

Smith, C. (1974). Concordance in twins: methods and interpretation. American fournal of Human Genetics, 26, 454-466.

Spitz, L. (1974). Congenital hypertrophic pyloric stenosis in triplets. Archives of Disease in Childhood, 49, 325.

Welsh, J. B. (1951). Pyloric stenosis in identical twins. Fournal of the Indiana State Medical Association, 44, 762.

William, H. J. (1963). Hypertrophic pyloric stenosis: brief review and report in premature twins. American fournal of Roentgeno- $\overrightarrow{0}$ $\log y, 89,1042-1047$. 